\title{
Excited State Dynamics of Curcumin and Solvent Hydrogen Bonding ${ }^{\dagger}$
}

\author{
Ilseung Yang, ${ }^{\ddagger}$ Seung Min Jin, ${ }^{\S}$ Junhee Kang, ${ }^{\#}$ Venkatnarayan Ramanathan, ${ }^{\ddagger}$ \\ Hyung Min Kim, ${ }^{\S}$ Yung Doug Suh,,${ }^{\S}$ and Seong Keun Kim ${ }^{\ddagger}, \#, *$ \\ Department of Chemistry, Seoul National University, Seoul 151-747, Korea

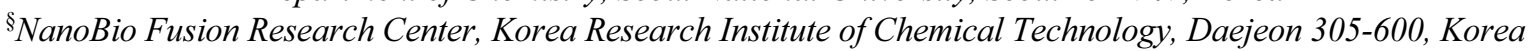 \\ ${ }^{\#}$ WCU Department of Biophysics and Chemical Biology, Seoul National University, Seoul 151-747, Korea \\ "E-mail: seongkim@snu.ac.kr \\ Received March 29, 2011, Accepted July 8, 2011
}

\begin{abstract}
Curcumin is a natural product with antioxidant, anti-inflammatory, antiviral and antifungal functions. As it is known that the excited state intramolecular hydrogen transfer of curcumin are related to its medicinal antioxidant mechanism, we investigated its excited state dynamics by using femtosecond transient absorption spectroscopy in an effort to understand the molecule's therapeutic effect in terms of its photophysics and photochemistry. We found that stronger intermolecular hydrogen bonding with solvents weakens the intramolecular hydrogen bonding and decelerates the dynamical process of the enolic hydrogen. Exceptions are found in methanol and ethylene glycol due to their nature as simultaneous hydrogen bonding donor-acceptor and high viscosity solvent, respectively.
\end{abstract}

Key Words : Curcumin, Excited state intramolecular hydrogen transfer, Transient absorption

\section{Introduction}

Curcumin, a yellow-orange natural pigment found in the rhizome of Curcuma longa, has drawn considerable interest in the past few decades due to its medicinal effects as an anti-inflammatory, anti-angiogenic, anti-cancer and antioxidant agent. ${ }^{1-4}$ The compound has been traditionally used not only as a main ingredient in Indian and Arabic cuisine for its distinctive flavor but also as a medicine for its therapeutic effects. ${ }^{5}$ Curcumin is also reported to prevent protein aggregation that often leads to diseases such as Alzheimer's and Parkinson's. ${ }^{6,7}$

Curcumin belongs to a group of $\beta$-diketonic compound which exhibits keto-enol tautomerism at room temperature. Several isomeric structures have been proposed but the predominant form in the ground state solution was found to be the keto-enol structure by NMR spectroscopy. ${ }^{8}$ The ketoenol form has a strong intermolecular hydrogen bonding interaction with the solvent through its hydrogen atoms. Excited state photophysics of curcumin has also been extensively studied, perhaps partly prompted by the enhancement of its medicinal effects upon excitation with light. ${ }^{9}$ The photogeneration of reactive oxygen species by curcumin, which is strongly dependent on the chemical nature of the solvent, has been speculated as the enhancement factor for its chemo-therapeutic activities. ${ }^{10-12}$ Several intensive studies have been reported about the connection between excited state intramolecular hydrogen transfer (ESIHT) and the medicinal properties of compounds such as hypericin and hypocrellin, both of which have a structural similarity

\footnotetext{
This paper is dedicated to Professor Eun Lee on the occasion of his ho-
} nourable retirement.

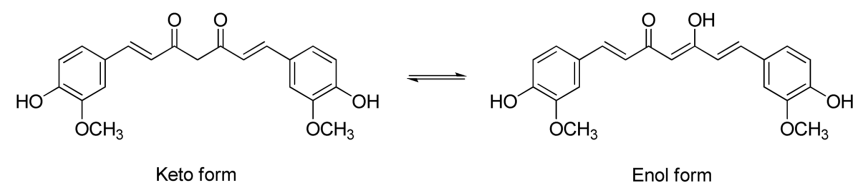

Figure 1. Chemical structures of curcumin, whose predominant form at room temperature is the enol form.

with curcumin. ${ }^{13-17}$ For such studies, a fundamental understanding on solvation dynamics and its effect on ESIHT is essential to elucidate the mechanisms involved.

Several groups have studied the excited state photophysics of curcumin in different kinds of solvents in the time domain of picoseconds to microseconds in organic solvents and micellar systems. ${ }^{11,18,19}$ Recently, Adhikary et al. studied the excited state photophysics of curcumin in protic solvent and surfactant micelles by using femtosecond fluorescence upconversion spectroscopy. ${ }^{20}$ They found that one of the decay time constants, i.e., the longer one, changed significantly when the enolic hydrogen of curcumin was deuterated. It was thus assigned to the ESIHT process, whereas the shorter time constant that did not change was thought to be associated with the process of solvation.

Most recently, Ghosh et al. used subpicosecond time-resolved transient absorption (TA) and fluorescence upconversion spectroscopy to study the excited state dynamics of curcumin, ${ }^{21}$ and reached basically the same conclusion as Adhikary et al.'s regarding the shorter time constant. For the longer time constant, however, they suggested that intermolecular hydrogen bonding between protic solvent and curcumin may sufficiently perturb the intramolecular hydrogen bonding of curcumin.

In view of these issues, we investigated in this paper the relationship between excited state dynamics of curcumin 
and its hydrogen bonding with various solvents using femtosecond TA spectroscopy.

\section{Experimental}

We used a typical TA setup to obtain femtosecond TA of curcumin in solution. ${ }^{22}$ A femtosecond pulse (130 fs, 120 $\mathrm{cm}^{-1}, 1 \mathrm{~W}, 1 \mathrm{kHz}$ ) centered at $800 \mathrm{~nm}$ was generated by a typical regeneratively-amplified Ti:Sapphire laser (Spitfire Pro, Spectra Physics) seeded by a femtosecond Ti:Sapphire oscillator (MaiTai, Spectra Physics) running at $80 \mathrm{MHz}$ repetition rate. The continuum light was generated by focusing the $800-\mathrm{nm}$ light by a planoconvex lens $(\mathrm{f}=10 \mathrm{~cm})$ into a $1 \mathrm{~mm}$-thick sapphire plate (Newport). To prevent chirping of the continuum light, gold-coated metal mirrors and concave mirrors were used. The pump pulses were generated using the second harmonic generator (TP-THG-F, Spectra Physics). The maximum power at the focal point was $100 \mu \mathrm{J}$ at $400 \mathrm{~nm}$. The pump and continuum lights had a diameter of $\sim 300 \mu \mathrm{m}(\mathrm{f}=70 \mathrm{~cm})$ and $\sim 100 \mu \mathrm{m}(\mathrm{f}=35 \mathrm{~cm})$, respectively. The polarization angle of pump pulses was controlled using a zero-order half-waveplate for $400 \mathrm{~nm}$ (QWPO-400-06-2-R, CVI). We kept the polarization angle of pump pulses at the magic angle of $54.7^{\circ}$. The optical time delay between the pump and continuum pulses was scanned by motorized translation stage (Daedal, Parker Hannifin Co.). The pump pulses were modulated using a synchronized optical chopper (MC1000, Thorlabs). The probe pulses of signal and reference beams were detected by photodiodes after the wavelength selection using polychromator (SP2300i, Princeton Instruments). The signal and reference intensity, which was generated using boxcar integrator (SR250, Stanford Research Systems), was divided using an analog processor (SR245, Stanford Research Systems) to compensate for the power fluctuation. The TA intensities were recorded using a lock-in amplifier (SR830, Stanford Research Systems). The cross correlation of femtosecond TA was $340 \pm 10 \mathrm{fs}$ for the $400 \mathrm{~nm}$ pump.

Curcumin (C1386, Aldrich) and HPLC-grade solvents (methanol, ethylene glycol, dimethyl formamide (DMF), acetonitrile, and chloroform, all from Aldrich) were purchased and used without further purification. UV/Vis absorption and fluorescence spectra were recorded by commercial instruments (Lambda 25, Perkin-Elmer and QM-3/2004SE, PTI, respectively) using a 1-cm path length quartz cell (111-QG, Hellma).

\section{Results and Discussion}

Table 1 lists the absorption and emission maxima of curcumin in various solvents, indicating a significant dependence on hydrogen bonding. The Stokes shift is noticeably smaller in non-hydrogen bonding solvent (chloroform) than in hydrogen bonding solvents (the top four). ${ }^{12,18,23-25}$ Solvents that can both donate and accept hydrogen bonds generally yield a larger shift than those that only accept hydrogen bond. ${ }^{25}$
Table 1. Spectral and chemical properties of the various solvents used to dissolve curcumin

\begin{tabular}{|c|c|c|c|c|c|}
\hline Solvent & H-bonding & $\begin{array}{l}\text { Absorption } \\
(\mathrm{nm})\end{array}$ & $\begin{array}{c}\text { Emission } \\
(\mathrm{nm})\end{array}$ & $\begin{array}{c}\text { Viscosity } \\
\left(\text { Poise, } 25^{\circ} \mathrm{C}\right)^{26}\end{array}$ & $\begin{array}{c}\text { Functional } \\
\text { group }\end{array}$ \\
\hline Methanol & $\begin{array}{l}\text { Donor- } \\
\text { Acceptor }\end{array}$ & 434 & 545 & 0.55 & $\mathrm{R}-\mathrm{OH}$ \\
\hline DMF & Acceptor & 429 & 526 & 0.80 & $R^{-C_{-1}}$ \\
\hline Acetonitrile & Acceptor & 417 & 514 & 0.34 & $R-C \equiv N$ \\
\hline $\begin{array}{l}\text { Ethylene } \\
\text { glycol }\end{array}$ & $\begin{array}{l}\text { Donor- } \\
\text { Acceptor }\end{array}$ & 435 & 541 & 13.76 & $\mathrm{HO}-\mathrm{R}-\mathrm{OH}$ \\
\hline Choloroform & $\begin{array}{c}\text { Non } \\
\text { H-bonding }\end{array}$ & 418 & 480 & 0.54 & $\mathrm{R}-\mathrm{Cl}$ \\
\hline
\end{tabular}

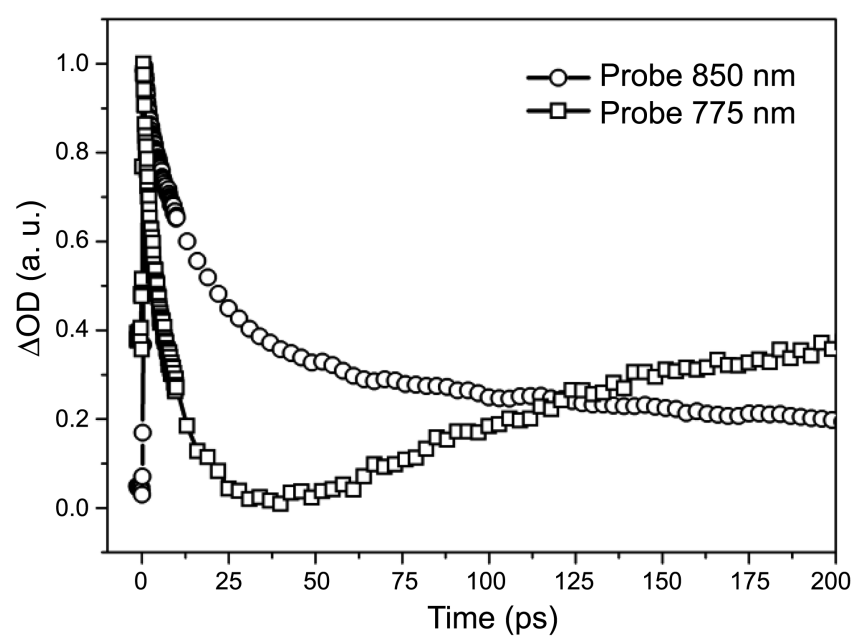

Figure 2. Temporal profile for TA of curcumin in methanol probed at two different wavelengths ( 775 and $850 \mathrm{~nm})$. At $775 \mathrm{~nm}$, both excited state absorption and stimulated emission are convoluted in the spectrum, while only the excited state absorption is observed at $850 \mathrm{~nm}$

In this work, we kept our probe wavelength at $850 \mathrm{~nm}$ to effectively measure the lifetime of only the excited state without being affected by stimulated emission. Figure 2 shows TA signal of curcumin in methanol at the probe wavelengths of 775 and $850 \mathrm{~nm}$. Temporal profile at $775 \mathrm{~nm}$ shows what appears to be a "negative absorption" that results from stimulated emission.

Figure 3 shows the temporal profiles of photoexcited curcumin in various solvents at the pump and probe wavelengths of 400 and $850 \mathrm{~nm}$, respectively, at time intervals of $166 \mathrm{fs}$ up to $10 \mathrm{ps}$ and $2 \mathrm{ps}$ afterwards. We chose the solvents to compare the strength of their hydrogen bonding with curcumin and the excited state decay rate. The temporal profiles were fitted with a biexponential function, whose time constants are listed in Table 2.

In their earlier work, Adhikary et al. obtained a biexponential decay for hydrogen bonding solvents (methanol and ethylene glycol) but only a single exponential decay for nonhydrogen bonding solvent (chloroform). ${ }^{20}$ They interpreted it as reflecting the absence of active solvation process with solvents of non-hydrogen bonding character. The time 


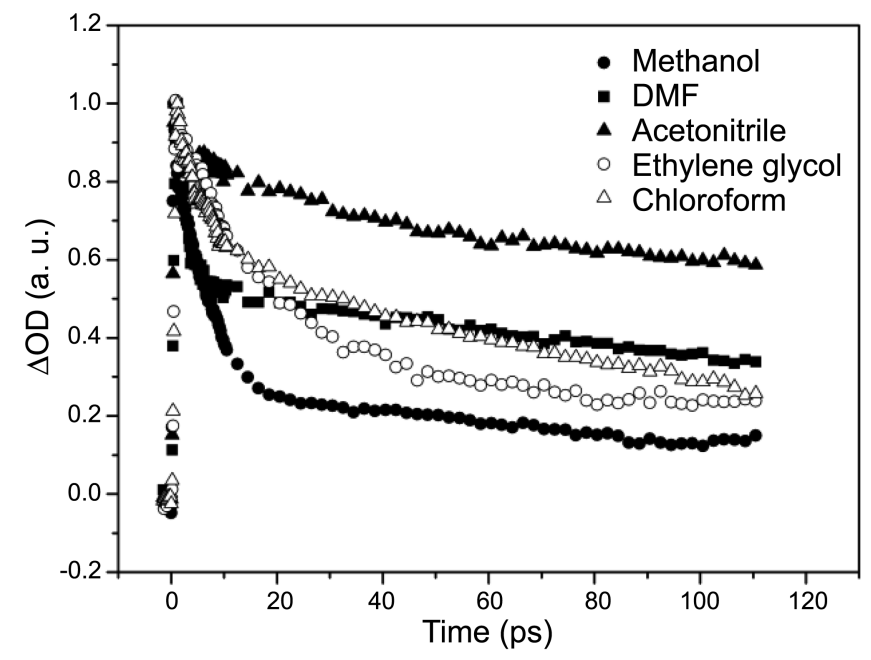

Figure 3. Temporal profile for TA of curcumin in various solvents.

constant of $130 \mathrm{ps}$ for their single exponential decay was assigned to ESIHT.

In our case, however, we found a biexponential decay for chloroform as well as all other solvents. Lack of hydrogen bonding does not necessarily preclude active solvation since there are other intermolecular interactions at play such as dipole-dipole interaction, and we think that our biexponential decay in chloroform indicates both solvation and ESIHT. From Table 2, we note that our shorter time constant $\tau_{1}$ for all solvents (regardless of their hydrogen bonding nature) is comparable to the time constant for solvation dynamics of coumarin 153 of Horng et $\mathrm{al}^{26}$ Since the dipole moment of curcumin $(\sim 6.1 \mathrm{D})$ is comparable to that of coumarin 153 $(\sim 8 \mathrm{D})$, we find this to be reasonable.

For the longer time constant, we note from Fig. 3 and Table 2 that a non-hydrogen bonding solvent (chloroform) gives a significantly shorter decay time constant $\tau_{2}$ than hydrogen bonding solvents. It is to be noted that a very fast decay of curcumin in non-hydrogen bonding solvents such as cyclohexane and 1,4-dioxane (each with a time constant of 44 and $55 \mathrm{ps)}$ ) in earlier studies ${ }^{18,21}$ was attributed to the fact that these solvents do not form intermolecular hydrogen bond with curcumin that would have weakened its (a)

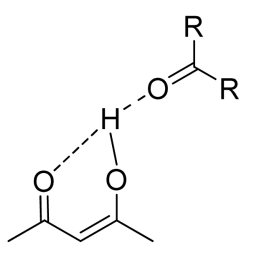

(b)

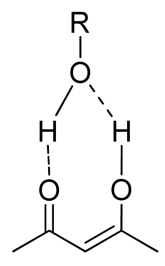

Figure 4. Schematic diagram showing the formation of intermolecular hydrogen bonding between curcumin and (a) hydrogen accepting solvents (b) hydrogen donating and accepting solvent.

intramolecular hydrogen bond, which would decrease the rate of its decay through ESIHT.

We also note that our longer time constant $\tau_{2}$ is largest in DMF and acetonitrile, which have a stronger hydrogen bonding capability than chloroform. This is because formation of intermolecular hydrogen bonding would weaken the original intramolecular hydrogen bond and also partially "lock" the enolic hydrogen involved in the intramolecular hydrogen transfer (as schematically shown in Fig. 4(a)), which acts as the main decay channel for the longer time constant. If this were the prevailing factor in determining $\tau_{2}$, methanol would have resulted in an even longer $\tau_{2}$ since its hydrogen bonding capability is the largest of all solvents we used. In reality, however, methanol gives a rather short $\tau_{2}$ because it can act as both hydrogen bonding donor and acceptor, as schematically shown in Fig. 4 (b). Such a structure would provide an indirect pathway for ESIHT and therefore partially compensate for the decrease in the decay rate based solely on intermolecular hydrogen bonding model. Ethylene glycol with its exceptionally high viscosity is a notable exception to the above discussion.

There seems that a general correlation exists between the decay time constant and the strength of intermolecular hydrogen bonding. In general, stronger intermolecular hydrogen bonding appears to decelerate the intramolecular transfer of the enolic hydrogen represented by the longer time constant for decay, but certain solvents can make up for the decrease in its rate by providing another, solventmediated, ESIHT pathway. These effects need to be carefully addressed to relate the strength of hydrogen bonding to the decay time constant via ESIHT.

Table 2. Time constants associated with the relaxation of the excited state of curcumin and coumarin 153 in various solvents

\begin{tabular}{|c|c|c|c|c|c|c|c|}
\hline \multirow[t]{3}{*}{ Solvent } & \multirow{2}{*}{\multicolumn{2}{|c|}{$\begin{array}{c}\text { Hydrogen } \\
\text { bonding strength }^{27}\end{array}$}} & \multirow{3}{*}{$\begin{array}{c}\text { Coumarin } \\
\text { Horng et al. }^{26} \\
<\tau> \\
(\mathrm{ps})^{25}\end{array}$} & \multicolumn{4}{|c|}{ Curcumin } \\
\hline & & & & \multicolumn{2}{|c|}{ Ghosh et al. ${ }^{21}$} & \multicolumn{2}{|c|}{ This work } \\
\hline & $\alpha$ & $\beta$ & & $\begin{array}{c}\langle\tau\rangle_{\text {solv }} \\
\text { (ps) }\end{array}$ & $\begin{array}{c}\tau_{3} \\
(\mathrm{ps})\end{array}$ & $\begin{array}{c}\tau_{1} \\
(\mathrm{ps})\end{array}$ & $\begin{array}{c}\tau_{2} \\
(\mathrm{ps})\end{array}$ \\
\hline Methanol & 0.98 & 0.66 & 5.0 & 6.9 & 130 & 6.26 & 172 \\
\hline DMF & 0.00 & 0.69 & 2.0 & 1.6 & 155 & 2.08 & 240 \\
\hline Acetonitrile & 0.19 & 0.40 & 0.26 & 0.44 & 300 & 0.9 & 255 \\
\hline Ethylene glycol & 0.91 & 0.52 & 15.3 & 10.5 & 231 & 16.25 & 333 \\
\hline Choloroform & 0.20 & 0.10 & 2.8 & & & 4.83 & 129 \\
\hline
\end{tabular}

$\alpha$ : hydrogen donating strength; $\beta$ : hydrogen accepting strength 


\section{Conclusions}

We investigated the excited state dynamics of curcumin in various solvents. All our temporal profiles showed a biexponential decay. We studied the effect of intermolecular hydrogen bonding strength between various solvents and curcumin on the excited state relaxation dynamics. We found that intermolecular hydrogen bonding with solvents weakens the intramolecular hydrogen bonding in the keto/ enol unit and "locks" the enolic hydrogen, thereby decelerating the transfer of the enolic hydrogen. Exceptions are found in methanol due to its nature as simultaneous hydrogen bonding donor-acceptor and ethylene glycol due to its high viscosity.

Acknowledgments. We would like to thank Professor Eun Lee of Seoul National University for years of encouragement and support of our research on molecular spectroscopy of biological and medicinal compounds such as the title molecule of this paper. This work was supported by the Chemical Genomics Grant (M1052602000208N2602-00210), the Star Faculty Program (KRF-2005084-C00017), and the World Class University Program (R31-2010-100320) of the National Research Foundation of Korea.

\section{References}

1. Ruby, A. J.; Kuttan, G.; Babu, K. D.; Rajasekharan, K. N.; Kuttan, R. Cancer Lett. 1995, 94, 79-83.

2. Lantz, R. C.; Chen, G. J.; Solyom, A. M.; Jolad, S. D.; Timmermann, B. N. Phytomedicine 2005, 12, 445-452.

3. Aggarwal, B. B.; Kumar, A.; Bharti, A. C. Anticancer Res. 2003, 23, 363-398.

4. Shi, M. X.; Cai, Q. F.; Yao, L. M.; Mao, Y. B.; Ming, Y. L.; Ouyang, G. L. Cell. Biol. Int. 2006, 30, 221-226.

5. Goel, A.; Kunnumakkara, A. B.; Aggarwal, B. B. Biochem Pharmacol. 2008, 75, 787-809.

6. Yang, F. S.; Lim, G. P.; Begum, A. N.; Ubeda, O. J.; Simmons, M.
R.; Ambegaokar, S. S.; Chen, P. P.; Kayed, R.; Glabe, C. G.; Frautschy, S. A.; Cole, G. M. J. Biol. Chem. 2005, 280, 5892-5901.

7. Masuda, M.; Suzuki, N.; Taniguchi, S.; Oikawa, T.; Nonaka, T.; Iwatsubo, T.; Hisanaga, S.; Goedert, M.; Hasegawa, M. Biochemistry-Us 2006, 45, 6085-6094.

8. Payton, F.; Sandusky, P.; Alworth, W. L. J. Nat. Prod. 2007, 70, 143-146.

9. Bruzell, E. M.; Morisbak, E.; Tonnesen, H. H. Photoch. Photobio. Sci. 2005, 4, 523-530.

10. Das, K. C.; Das, C. K. Biochem. Bioph. Res. Co. 2002, 295, 62-66.

11. Gorman, A. A.; Hamblett, I.; Srinivasan, V. S.; Wood, P. D. Photochem. Photobiol. 1994, 59, 389-398.

12. Chignell, C. F.; Bilski, P.; Reszka, K. J.; Motten, A. G.; Sik, R. H.; Dahl, T. A. Photochem. Photobiol. 1994, 59, 295-302.

13. Das, K.; English, D. S.; Petrich, J. W. J. Phys. Chem. A 1997, 101, 3241-3245.

14. English, D. S.; Das, K.; Ashby, K. D.; Park, J.; Petrich, J. W.; Castner, E. W. J. Am. Chem. Soc. 1997, 119, 11585-11590.

15. English, D. S.; Das, K.; Petrich, J. W. Abstr. Pap. Am. Chem. S 1997, 213, 405-PHYS.

16. Petrich, J. W. Int. Rev. Phys. Chem. 2000, 19, 479-500.

17. Smirnov, A. V.; Das, K.; English, D. S.; Wan, Z.; Kraus, G. A.; Petrich, J. W. J. Phys. Chem. A 1999, 103, 7949-7957.

18. Khopde, S. M.; Priyadarsini, K.; Palit, D. K.; Mukherjee, T. Photochem. Photobiol. 2000, 72, 625-631.

19. Ortica, F.; Rodgers, M. A. J. Photochem. Photobiol. 2001, 74, 745-751.

20. Adhikary, R.; Mukherjee, P.; Kee, T. W.; Petrich, J. W. J. Phys. Chem. B 2009, 113, 5255-5261.

21. Ghosh, R.; Mondal, J. A.; Palit, D. K. J. Phys. Chem. B 2010, 114, 12129-12143.

22. Jin, S. M.; Lee, Y. J.; Yu, J. W.; Kim, S. K. B. Korean Chem. Soc. 2004, 25, 1829-1832.

23. Nardo, L.; Paderno, R.; Andreoni, A.; Masson, M.; Haukvik, T.; Tonnesen, H. H. Spectrosc-Int. J. 2008, 22, 187-198.

24. Bong, P. H. B. Korean Chem. Soc. 2000, 21, 81-86.

25. Priyadarsini, K. I. J. Photoch. Photobio. C 2009, 10, 81-95.

26. Horng, M. L.; Gardecki, J. A.; Papazyan, A.; Maroncelli, M. J. Phys. Chem-Us 1995, 99, 17311-17337.

27. Riddick, J. A.; Bunger, W. B.; Sakano, T. K. Organic Solvents: Physical Properties and Methods of Purification; John Wiley \& Sons, Inc.: New York, 1986.

28. Marcus, Y. J Solution Chem. 1991, 20, 929-944. 